\title{
A new model to evaluate Raf signaling in hematopoietic cells
}

\author{
KEIKO OKUDA
}

\author{
Department of Molecular Diagnostics and Therapeutics, Kyoto Prefectural University \\ of Medicine Graduate School of Medical Science, Kyoto, Japan
}

Received April 8, 2013; Accepted May 29, 2013

DOI: $10.3892 /$ ijo.2013.2002

\begin{abstract}
The Raf/MEK/ERK pathway is thought to be critical in mediating cell survival and proliferation by cytokine receptors. However, the exact contribution of Raf is complex and not well understood. A better understanding of Raf signaling is important because of the recent observation that B-Raf is frequently mutated in various human cancers. We have generated a new model system that activates Raf directly by linking the extracytoplasmic and transmembrane domains of the erythropoietin receptor (EPOR) with the catalytic domain of Raf (CR3). This synthetic oncogene in which dimerization can be controlled by an exogenous ligand, is fixed at the cellular membrane, while the endogenous Raf is normally activated by binding with Ras. The chimeric receptor EPOR/CR3 was stably expressed in $\mathrm{Ba} / \mathrm{F} 3$ cells which lack EPO receptors. Although the lines remained dependent on IL-3 for proliferation, EPO treatment reduced the rate of cell death in the absence of IL-3. Also, EPO was synergistic with sub-optimal concentrations of IL-3 in inducing long-term cell proliferation, but did not augment proliferation of cells cultured with full concentrations of IL-3. EPO induced a rapid activation of ERK and also phosphorylation of endogenous Raf. It also induced tyrosine phosphorylation of several cellular proteins. The MEK1 inhibitor PD98059 reduced EPO-induced tyrosine phosphorylation, suggesting these substrates are downstream of MEK kinase. Interestingly, PD98059 also reduced the phosphorylation of endogenous Raf, indicating there is a positive feedback mechanism in Raf activation. We conclude that Raf can be activated by a mechanism that induces clustering at the cell membrane, and that this leads directly to activation of MEK and ERK. This EPOR/CR3 system may serve as a useful model to evaluate the unknown Raf kinase pathway and the effects of signal transduction inhibitors for Raf as a target.
\end{abstract}

Correspondence to: Dr Keiko Okuda, Department of Molecular Diagnostics and Therapeutics, Kyoto Prefectural University of Medicine Graduate School of Medical Science, 465 Kajii-cho, Kawaramachi-Hirokoji, Kamigyo-ku, Kyoto 602-8566, Japan E-mail: keiko_o@koto.kpu-m.ac.jp

Key words: Raf, chimeric receptor, signal transduction, transforming activity

\section{Introduction}

The Raf-MEK-ERK signal transduction pathway is a conserved RAS-activated protein kinase cascade that regulates cell growth, proliferation, and differentiation in response to growth factors, cytokines, and hormones (1-3). Activation of Raf in vivo occurs at the plasma membrane and depends on an association with Ras GTP. Subsequently, the serine/threonine kinase of Raf activates the dual-specificity kinases MEK1/2, which in turn phosphorylate the ERK1/2 kinases (MAPK) at both threonine and tyrosine residues. However, the mechanism of Raf activation and the exact contribution of Raf in signaling are not completely understood. Raf kinases (A-Raf, B-Raf and Raf-1) have three conserved regions, CR1, CR2 and CR3 (4). The CR1 region consists of two domains, a Ras-binding domain and a cysteine-rich domain, both of which bind Ras (5). The CR2 region is rich in serines and threonines and is the site of several phosphorylation events in Raf-1 (6). The CR3 region is the kinase domain that is regulated by phosphorylation events. The viral oncogenic form of Raf, $\mathrm{v}$-Raf, consists of a viral gag sequence at the $\mathrm{N}$-terminus followed by the whole catalytic domain of Raf. The constitutive activity and high transforming potential of $\mathrm{v}$-Raf most likely results from loss of the autoinhibitory $\mathrm{N}$-terminal region combined with targeting to the plasma membrane (7-9).

Mutations in one Raf gene, B-Raf were recently observed in a high proportion of malignant melanoma (66\%), and in a moderate to high fraction of colorectal, ovarian, and papillary thyroid carcinomas $(10,11)$. Most of these mutations were shown to be within the kinase domain and mutated B-Raf proteins had elevated kinase activity, constitutively stimulated ERK activity in vivo independent of RAS, and potently transformed NIH3T3 cells (12). As Raf is a serine/threonine kinase that can originate the oncogenic signaling pathway in human cancer, a better understanding of Raf signaling is important and may provide new therapeutic opportunities in cancer.

In an effort to develop a new model system in which Raf protein kinase activity could be directly and conditionally activated in mammalian cells at the cellular membrane, gene fusion between the catalytic domain (CR3) of Raf and the extracellular ligand-binding domain of the erythropoietin receptor (EPOR) was constructed (EPOR/CR3). Erythropoietin (EPO) is known to activate its receptor by dimerization and we previously demonstrated that fusion of the extracellular domain of the EPOR to c-ABL rendered the activity of ABL's tyrosine kinase dependent on the addition 
of EPO in cells expressing the chimeric receptor (13). This study documents the first example of the harnessing of Raf kinase activity by a similar strategy. In the resulting molecule as a transmembrane receptor, the CR3 domain of Raf was tethered to the cell membrane, where endogenous Raf is normally activated by binding with Ras (14). The cDNA encoding this structure was expressed in a non-leukemic, factor-dependent, murine hematopoietic cell line, $\mathrm{Ba} / \mathrm{F} 3$ and the biochemical and biological activities of this molecule were then examined.

\section{Materials and methods}

Plasmid constructs. A cDNA encoding the ligand-binding domain of the EPO receptor (EPOR) fused to the kinase domain of Raf-1 (CR3) was generated by PCR using cDNAs for the murine EPOR and human Raf-1 as templates. The EPOR/CR3 junction was made in frame at leucine residue 277 of the EPOR, and at serine residue 316 of Raf-1. The resulting protein contained the transmembrane domain and 6 additional intracellular amino acids of the EPOR, followed by Raf-1 CR3. Previous studies have shown that an EPOR truncated at tryptophan 282 is non-functional and can not activate known signaling pathways (15). The PCR product was ligated into the expression vector pcDNA3 and transfected into $\mathrm{Ba} / \mathrm{F} 3$ cells as described below.

Cell lines and cell culture. $\mathrm{Ba} / \mathrm{F} 3$ cells were maintained in RPMI-1640 medium supplemented with $10 \%$ (vol/vol) FCS and $10 \%$ conditioned medium from WEHI-3B cells as a source of murine IL-3. The parental $\mathrm{Ba} / \mathrm{F} 3$ cells used in these experiments do not express detectable endogenous EPO receptors, nor do they proliferate in response to EPO.

Plasmid pcDNA3 EPOR/CR3, which contains a neomycin resistance gene, was transfected into $\mathrm{Ba} / \mathrm{F} 3$ cells using electroporation with a gene pulser (Bio-Rad, Hercules, CA). Cells were grown for $48 \mathrm{~h}$ in $10 \%$ FCS RPMI-1640, supplemented with $10 \%$ WEHI conditioned medium before the addition of $\mathrm{G} 418(1 \mathrm{mg} / \mathrm{ml})$. After 2 to 3 weeks of initial selection, individual subclones were obtained by limiting dilution. All cells were grown at $37^{\circ} \mathrm{C}$ in a $5 \% \mathrm{CO}_{2}$ humidified incubator.

Flow cytometric analysis of EPO receptor expression. $\mathrm{Ba} / \mathrm{F} 3$ cells and their subclones were washed and resuspended in RPMI-1640 medium. Cells $\left(1.0 \times 10^{6}\right)$ were incubated with a saturating concentration of a protein A-purified polyclonal antiserum directed against the extracytoplasmic domain of human EPOR (kindly provided by Dr Alan D. Andrea, Dana-Farber Cancer Institute, Boston, MA) (16) in a 1:200 dilution in a total volume of $200 \mu 1$. Cells were washed with PBS and stained with an FITC-conjugated anti-rabbit second antibody. Cells were analyzed with a flow cytometer (FACS Calibur). Non-immune rabbit serum was used as a negative control.

Antibodies. Antiphosphotyrosine monoclonal antibody (4G10) was provided by Dr Brian Druker (University of Oregon Health Sciences Center, Portland, OR). Two rabbit polyclonal anti-Raf- 1 antibodies, which recognize either amino acids 637-648 mapping at the carboxy-terminus (CR3) or amino acids 180-250 mapping within an internal region (CR1-2) of Raf-1, and anti-CBL antibodies were purchased from Santa Cruz Biotechnology Inc. (Santa Cruz, CA). Anti-Shc antibody was purchased from Transduction Laboratories Inc. (Lexington, KY). Anti-phospho-p44/42 MAP kinase antibody which recognizes the MAP kinase only when phosphorylated at threonine 202 and tyrosine 204, was purchased from Cell Signaling Technology Inc. (Beverly, MA). Anti-phospho STAT polyclonal antibodies recognizing each of only tyrosine phosphorylated STAT 1, 3 or 5 were provided by Dr David Frank (Dana-Farber Cancer Institute, Boston, MA).

Immunoblot analysis and immunoprecipitation. Cells were deprived of growth factors by culturing in medium containing $10 \%$ FCS in RPMI-1640, and were then stimulated with either human recombinant EPO or murine recombinant IL-3 as indicated in each experiment. Immunoblot analysis and immunoprecipitation were performed using standard methods, and proteins were separated on a 7.5 or $9.0 \%$ SDS polyacrylamide gel. The MEK1 inhibitor PD98059 was purchased from Calbiochem Inc. (La Jolla, CA) and was used for the experiments indicated.

Proliferation and viability assays. The number of viable cells was determined by trypan blue, and viable cells were counted using a hemacytometer. Cell viability was reported as a percentage of the total cells.

Transwell migration assay. The membranes of Transwell chambers $(8 \mu \mathrm{m}$ pore-size polycarbonate membrane, Corning Coster Corp., Cambridge, MA) were coated with soluble human fibronectin (Gibco-BRL, Gaitherburg, MD) at a concentration of $10 \mu \mathrm{g} / \mathrm{ml}$ overnight at $4^{\circ} \mathrm{C}$. The lower chamber contained $600 \mu \mathrm{l}$ of RPMI-1640 containing $0.5 \%$ (w/v) bovine serum albumin (BSA). A total of $0.2 \times 10^{6}$ cells in $100 \mu \mathrm{l}$ of RPMI-1640 containing 0.5\% (w/v) BSA with or without EPO and/or IL-3 were placed into the upper chamber and cultured at $37^{\circ} \mathrm{C}$ for $5 \mathrm{~h}$. The same experiments were performed in the presence or absence of PD98059. After $5 \mathrm{~h}$, cells in the lower compartment were counted by trypan blue exclusion. The spontaneous Transwell migration of cells was expressed as a migration index (number of migrating cells treated with the indicated cytokine divided by the number of migration cells left untreated).

\section{Results}

Construction and expression of EPOR/CR3 fusion proteins. To examine the specific role of Raf in signal transduction, we used an erythropoietin-induced dimerization method (13). The chimeric construct possess only a kinase domain (CR3) and not a CR1 or CR2 fragment of Raf as shown in a schematic diagram in Fig. 1A, so that the molecule is fixed at the cellular membrane without binding to endogenous Ras in this system.

The IL-3-dependent $\mathrm{Ba} / \mathrm{F} 3$ cell line was transfected with EPOR/CR3 cDNA in an expression vector as described in Materials and methods. The expression of the chimeric receptor in a polyclonal subline was confirmed by both immunofluorescent cell staining with an anti-EPOR antibody (Fig. 1B) and immunoblot analysis with an anti-Raf antibody (Fig. 1C). 
A

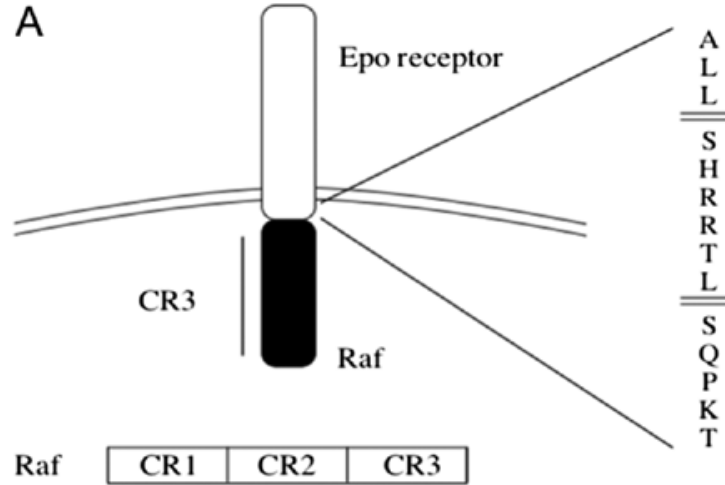

B

trans

memb

Epo R

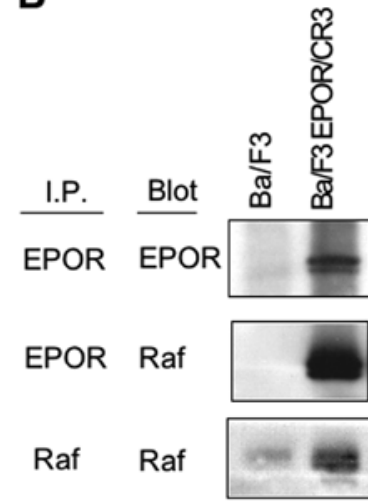

C
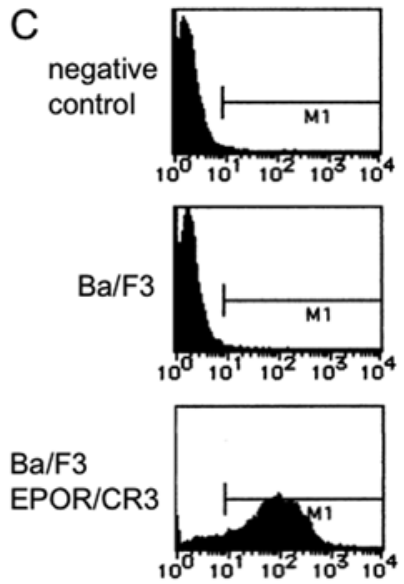

Figure 1. A model of the EPOR/CR3 chimeric molecule. (A) The EPOR/CR3 construct used in this study is summarized. cDNA encoding the ligand-binding domain of the EPO receptor (EPOR) fused to the Raf CR3 domain was generated by PCR using cDNAs for the murine EPOR and human Raf as templates. The resulting protein contains the transmembrane domain of the EPOR and 6 additional intracellular amino acids of the EPOR, followed by Raf CR3. CR3, catalytic domain of Raf. Endogenous Raf is located in the cytoplasm. The protein expression was confirmed by (B) immunoblot analysis and (C) immunophenotyping. The expression of a chimeric molecule was confirmed by immunoblot analysis after immunoprecipitation with either anti-EPOR or anti-Raf antibody. EPOR surface expression was detected using anti-EPOR for FACS analysis, which indicated that the chimeric receptor was expressed at the cell membrane.

Erythropoietin induces the phosphorylation of EPOR/CR3 and several other cellular proteins in EPOR/CR3 expressing $B a / F 3$ cells. To compare the signal transduction pathway induced by growth factor stimulation, the tyrosine phosphorylation status of the cellular proteins were first examined by immunoprecipitation followed by western blot analysis with the anti-phosphotyrosine antibody (4G10). As shown in Fig. 2, the addition of EPO also induced the tyrosine phosphorylation of other cellular proteins of approximately 150, 130, 110, 95, 60 and $42 \mathrm{kD}$ in EPOR/CR3 cells. EPO did not induce tyrosine phosphorylation of cellular proteins in parental $\mathrm{Ba} / \mathrm{F} 3$ cells not expressing EPOR/CR3 (data not shown). Since Raf/MEK/ERK is one of the well known major signal transduction pathways induced by growth factor stimulation, changes in the biochemical or biological phenotype using the MEK1 inhibitor PD98059 were observed in each experiment. PD98059 reduced EPO and/ or IL-3-induced the tyrosine phosphorylation of many, but not all, cellular proteins, as shown in the figure.

Several proteins that are tyrosine phosphorylated by IL-3 stimulation have been previously identified. Therefore, we asked if activation of the EPOR/CR3 kinase resulted in the tyrosine phosphorylation of any of the same substrates. The molecules that were tyrosine phosphorylated after EPO-induced activation of EPOR/CR3 fusion proteins were identified by immunoprecipitation with an anti-phosphotyrosine antibody followed by specific antibody blotting. According to their molecular weight, we first examined CBL, STAT1, 3, 5, PI3K, Shc and ERK1/2, which are known to be tyrosine phosphorylated by IL-3 stimulation in Ba/F3 cells. The activated EPOR/CR3 protein was found to induce the tyrosine phosphorylation of CBL and ERK1/2, but not STAT1, 3, 5, PI3K or Shc (Fig. 3). The tyrosine phosphorylation of both CBL and ERK1/2 was abolished by PD98045, indicating that phosphorylation was due to the activated MEK kinase. Since Raf itself is a serine/threonine kinase, the phosphorylation state of endogenous Raf-1 and Paxillin was focused on next as the substrate. Endogenous Raf-1 was collected by immunoprecipitation using an anti-Raf

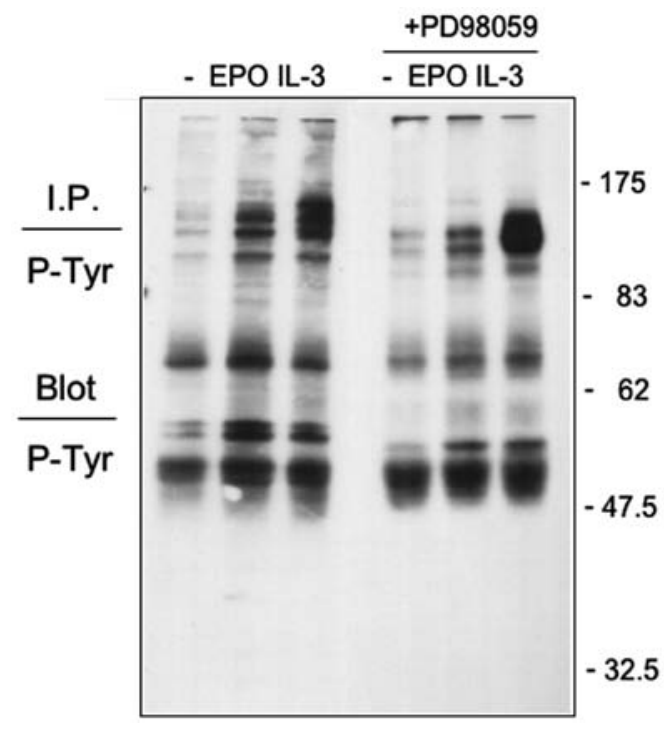

Figure 2. EPO-induced protein tyrosine phosphorylation in the EPOR/CR3 cells. Cells were factor-deprived for $4 \mathrm{~h}$, pretreated with $50 \mu \mathrm{M}$ of PD98059 (+) or without PD98059 (-) (DMSO alone) for $3 \mathrm{~h}$, and were then stimulated with either $5 \mathrm{U} / \mathrm{ml}$ EPO or $1 \mathrm{ng} / \mathrm{ml} \mathrm{IL-3} \mathrm{for} 15 \mathrm{~min}$. Proteins were separated in a SDS-PAGE, and changes in protein tyrosine phosphorylation were detected by immunoblot analysis with a monoclonal antiphosphotyrosine antibody (4G10).

antibody, which recognized the CR1-2 region of Raf-1. As shown in Fig. 4, IL-3 and EPO stimulation of factor-deprived EPOR/CR3 cells caused a rapid shift to a slower migrating form of endogenous Raf-1 and Paxillin. These results indicated that EPO induced the serine/threonine phosphorylation of endogenous Raf and Paxillin, as indicated by a mobility shift on SDS-polyacrylamide gels, while there was little or no induction, if any, in the phosphorylation of tyrosine detected with both proteins. These shifts in both proteins were principally or exclusively due to MEK/ERK kinase through Raf activation and not to a direct effect of Raf kinase because the treatment of cells with PD98059 reduced this band to comigrate with that from 


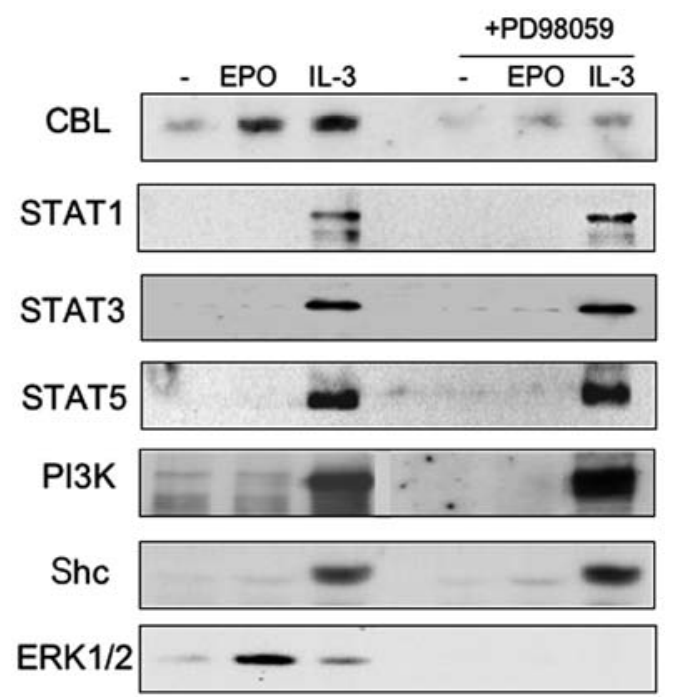

Figure 3. Tyrosine phosphorylation of signaling proteins in EPOR/CR3 cells. The phosphorylation state of individual proteins was evaluated by immunoprecipitation using an anti-phosphotyrosine antibody followed by blotting with specific antibodies. The prominent targets of the wild-type IL-3 receptor, Shc, STAT1, 3 and 5 were not tyrosine phosphorylated by the EPOR/CR 3 receptor with EPO stimulation. The tyrosine phosphorylation of ERK1/2 was eliminated by PD98059.

unstimulated cells. The phosphorylation of endogenous Raf-1 induced by EPO was reduced by PD98059, indicating a positive feedback mechanism in Raf activation. Overall, these results indicate that the EPOR/CR3 kinase has very low kinase activity in the absence of added EPO, and is rapidly and persistently activated after the addition of EPO.

Activation of the EPOR/CR3 in Ba/F3 cells by EPO enhances viability, but does not sustain strong proliferation by itself. Since Raf has been reported to play an essential role in the cell proliferation induced by various stimuli, the biological effects of activating Raf kinase in EPOR/CR3 Ba/F3 cells were examined.

To determine whether EPOR/CR3 fusion proteins had a growth-promoting function, changes in the total number of viable cells were analyzed in response to EPO. Cells were washed with depletion media and cultured in various concentrations of EPO or IL-3 for the indicated period. In response to EPO stimulation, a dose-dependent slower increase in the number of viable cells was observed in EPOR/CR3 Ba/F3 cells, but no effect was observed on control $\mathrm{Ba} / \mathrm{F} 3$ cells. Short term effects on growth are shown in Fig. 5. At EPO concentrations over $1 \mathrm{U} / \mathrm{ml}$, EPO maintained cell number and viability for several days, but did not cause significant proliferation. The maximum rate of EPO-induced proliferation of EPOR/CR3 cells was approximately 5-fold less in 4 days than that observed in the case of IL-3 stimulation, even with an optimal concentration of EPO $(5 \mathrm{U} / \mathrm{ml})$. In the absence of $\mathrm{EPO}, \mathrm{EPOR} / \mathrm{CR} 3 \mathrm{Ba} / \mathrm{F} 3$ cells died at the same rate as parental $\mathrm{Ba} / \mathrm{F} 3$ cells cultured in the absence of growth factor.

This result indicated that Raf activation could not sustain strong proliferation by itself in this system. The long-term cell viability of the EPOR/CR3 cells was then tested in the presence of EPO by trypan blue exclusion. Although virtually no cell was viable after $48 \mathrm{~h}$ of factor depletion, the presence

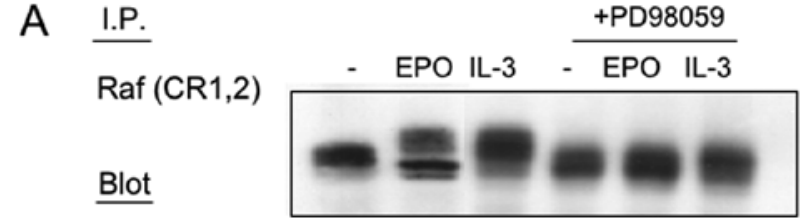

Raf (CR3)

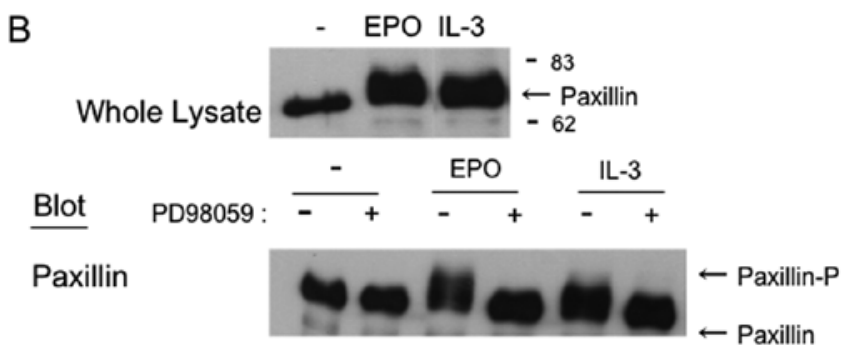

Figure 4. EPO induced phosphorylation of endogenous Raf-1 and Paxillin in EPOR/CR3. EPOR/CR3 cells were factor-deprived for $4 \mathrm{~h}$, pretreated with or without PD98059 $(50 \mu \mathrm{M})$ for $3 \mathrm{~h}$, and were then stimulated with either $5 \mathrm{U} / \mathrm{ml}$ EPO or $1 \mathrm{ng} / \mathrm{ml} \mathrm{IL-3} \mathrm{for} 15 \mathrm{~min}$. (A) The anti-Raf antibody, which was raised against the internal region (amino acids 180-250, CR1-2) of Raf-1 was used for immunoprecipitation in order to collect endogenous Raf-1. Immunoprecipitates were applied to a $7.5 \%$ gel and probed with anti-Raf-1 antibody. The slower migration of Raf-1, observed with EPO stimulation, was eliminated by PD98059. (B) The phosphorylation state of the cytoskeleton protein Paxillin was evaluated using whole cell lysates as another candidate for a serine/threonine kinase substrate. Paxillin immunoblot analysis demonstrated Paxillin electrophoretic mobility in EPOR/CR3 cells. Pre-treatment with PD98059 decreased Paxillin electrophoretic mobility induced by both EPO and IL-3, which indicates that MEK kinase was important for Paxillin phosphorylation.

of EPO maintained a small, but significant population of EPOR/CR3 cells viable for $>96 \mathrm{~h}$. After $240 \mathrm{~h}$ of culturing, no cell was viable, which indicates that EPOR/CR3 can sustain short-term, but not long-term proliferation or cell survival (data not shown).

EPO does not make cells hyper-responsive to IL-3, but is synergistic with a sub-optimal dose of IL-3 in inducing long-term cell proliferation. Some oncogenes have been reported to increase the sensitivity of progenitor cells to hematopoietic growth factors such as IL-3. We tested the effects of activating the EPOR/CR3 receptor on the sensitivity of $\mathrm{Ba} / \mathrm{F} 3$ cells to IL-3-induced proliferation. IL-3 dose-response curves were generated in the presence of a fixed concentration of EPO. Although EPO increased baseline proliferation in the absence of IL-3, it did not shift the dose-response curve (Fig. 6A). However, EPO induced long-term cell proliferation together with a sub-optimal concentration of IL-3 $(0.1 \mathrm{ng} / \mathrm{ml})$, as shown in Fig. 6B. PD98059 did not have an obvious effect on the long-term proliferation activity and viability-promoting activity induced by full and sub-optimal concentrations of IL-3 themselves, respectively. Interestingly, the viability-promoting activity of EPOR/CR3 was profoundly impaired by PD98059, but the ability to induce long-term proliferation with a sub-optimal concentration of IL-3 was sustained (Fig. 7).

These results suggest that activation of Raf in the context of this chimeric receptor does not result in synergy with the IL-3 receptor. However, there was an additive effect of EPOR/CR3 activation and IL-3 receptor activation. 
$\mathrm{Ba} / \mathrm{F3}$

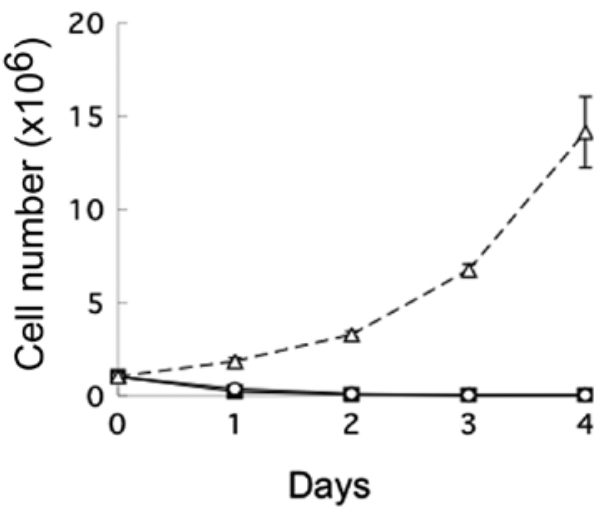

$\mathrm{Ba} / \mathrm{F} 3 \mathrm{EPOR} / \mathrm{CR} 3$

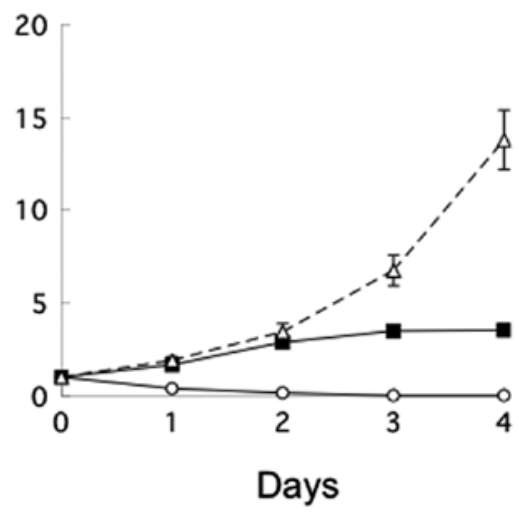

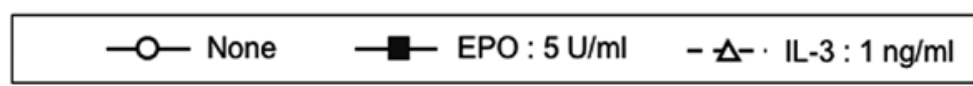

Figure 5. EPO induced viability in Ba/F3 EPOR/CR3 cells. Cells were washed 3 times and re-suspended at a density of $0.5 x 10^{6}$ cells/ml x 2 ml each in RPMI supplemented with $10 \%$ fetal calf serum. Cells were then cultured at $37^{\circ} \mathrm{C}$ with or without the indicated growth factor. The total number of viable cells was evaluated every $24 \mathrm{~h}$. The EPOR/CR3 cells remained dependent on IL-3 for proliferation as parental Ba/F3. The addition of EPO had no effect on parental Ba/F3 cells, while EPO treatment reduced the rate of cell death and induced weak proliferation in the absence of IL-3 in EPOR/CR3 lines.

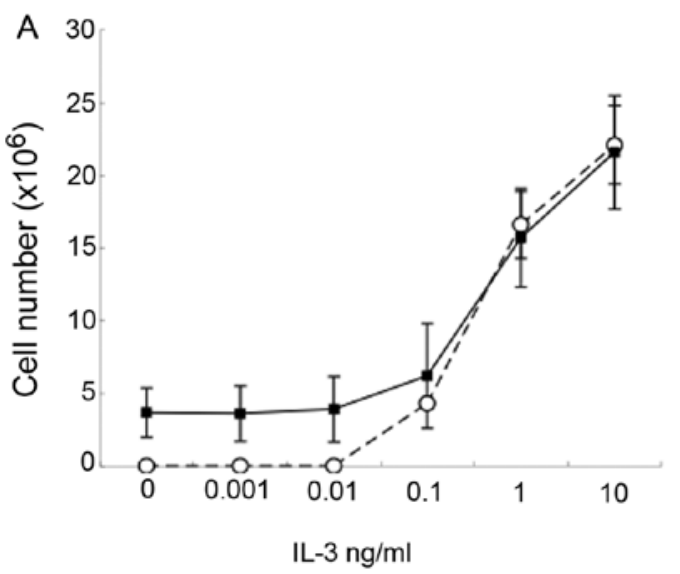

$-0-$-EPO $\rightarrow+$ EPO $5 \mathrm{U} / \mathrm{ml}$
B
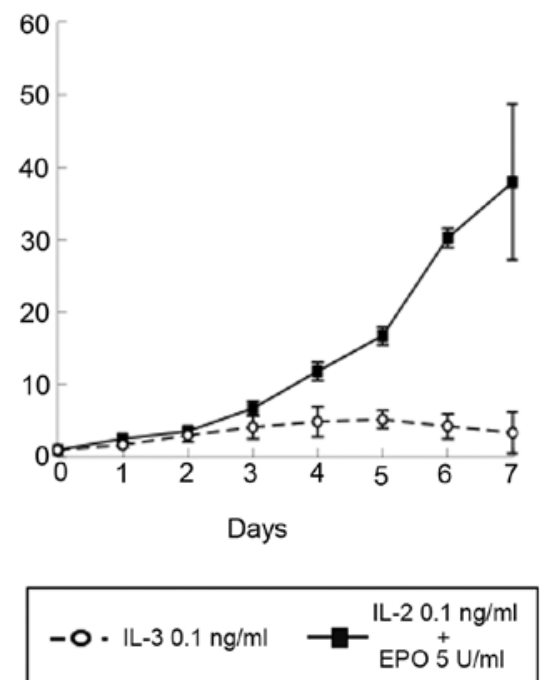

Figure 6. Effect of Raf kinase on IL-3-induced cell proliferation. (A) Cells were washed 3 times and re-suspended at a density of $0.5 \times 10^{6}$ cells/ml x 2 ml each in $10 \%$ FCS-RPMI. Cells were then incubated with the indicated concentration of IL-3, in the absence or presence of EPO. The total number of viable cells was evaluated every $24 \mathrm{~h}$ and the cell number on day 4 is reported in the figure. EPO did not augment the proliferation of cells cultured with full concentrations of IL-3. (B) The growth curve of cells with either a low dose of IL-3 alone or a low dose of IL-3 + EPO. This sub-optimal concentration of IL-3 could only maintain cell survival, while addition of EPO to EPOR/CR3 lines made the cells proliferate for culture periods greater than 3 weeks.

The EPOR/CR3 receptor enhances cell migration without affecting $\beta 1$ integrin expression levels. Hematopoietic cells spontaneously migrate on surfaces coated with extracellular matrix proteins when stimulated with hematopoietic growth factors or certain oncogenes. Migration assays were performed on these cells. As shown in Fig. 8, EPOR/CR3 expression enhanced spontaneous migration. PD98059 decreased migration activity, but did not abrogate it, indicating that there is another signal transduction pathway besides Raf/MEK/ERK that leads to migration activity.

The possibility that EPOR/CR3 expression augmented $\beta 1$-integrin surface expression was examined, however, no detectable effect was observed using FACS analysis with an anti- $\beta 1$-integrin antibody (data not shown).

\section{Discussion}

In the present study, a new model was made in order to analyze the mechanisms of Raf activation and its specific functions by constructing a chimeric molecule containing the ligand-binding domain of the EPOR and the kinase domain (CR3) of Raf. The resulting molecule EPOR/CR3, which functioned as a transmembrane receptor, could be artificially dimerized by erythropoietin (EPO). 

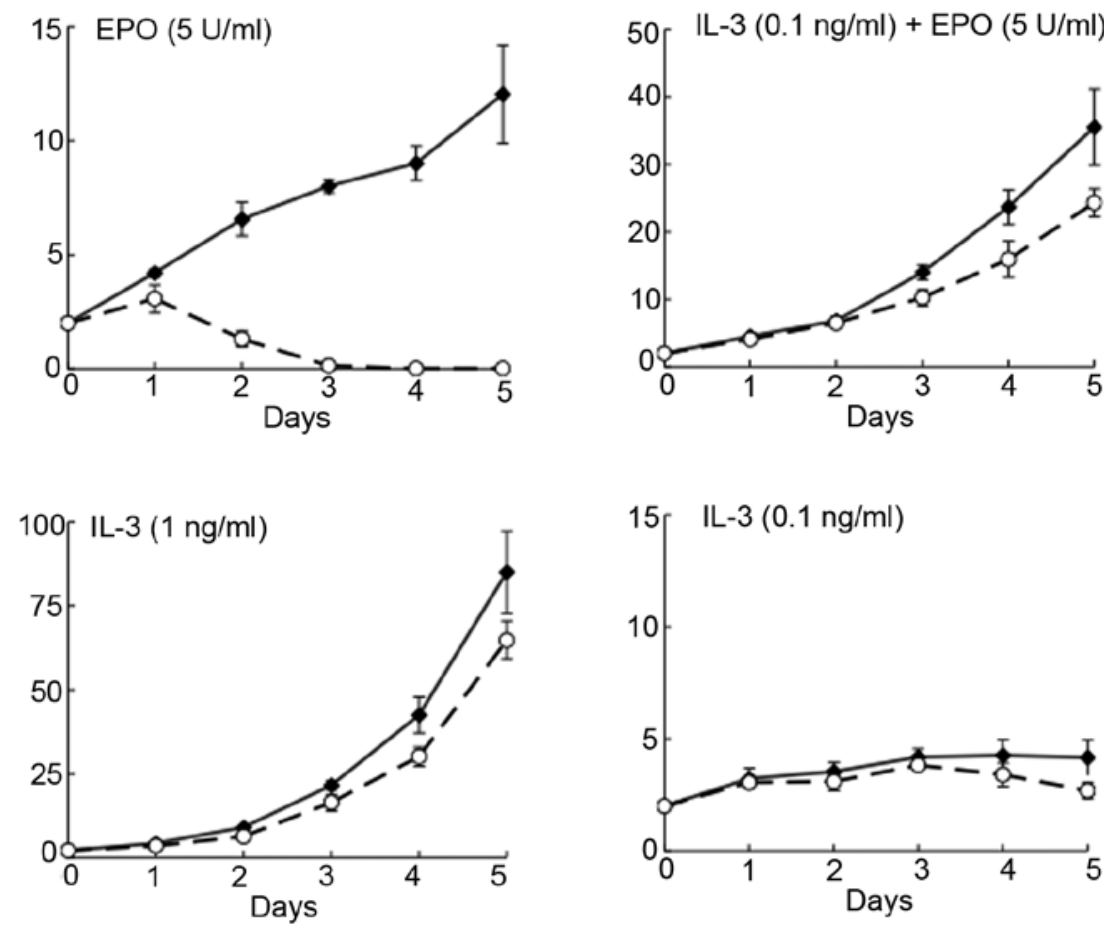

Figure 7. The synergistic effect of EPO with a low dose of IL-3 was not abolished by the MEK inhibitor PD98059. Cells were washed 3 times and re-suspended at a density of $0.5 \times 10^{6}$ cells $/ \mathrm{ml} \mathrm{x} 4 \mathrm{ml}$ each in $10 \%$ FCS-RPMI, and were then incubated with the indicated concentration of factors, in the absence or presence of $50 \mu \mathrm{M}$ PD98059. The total number of viable cells was evaluated every $24 \mathrm{~h}$.

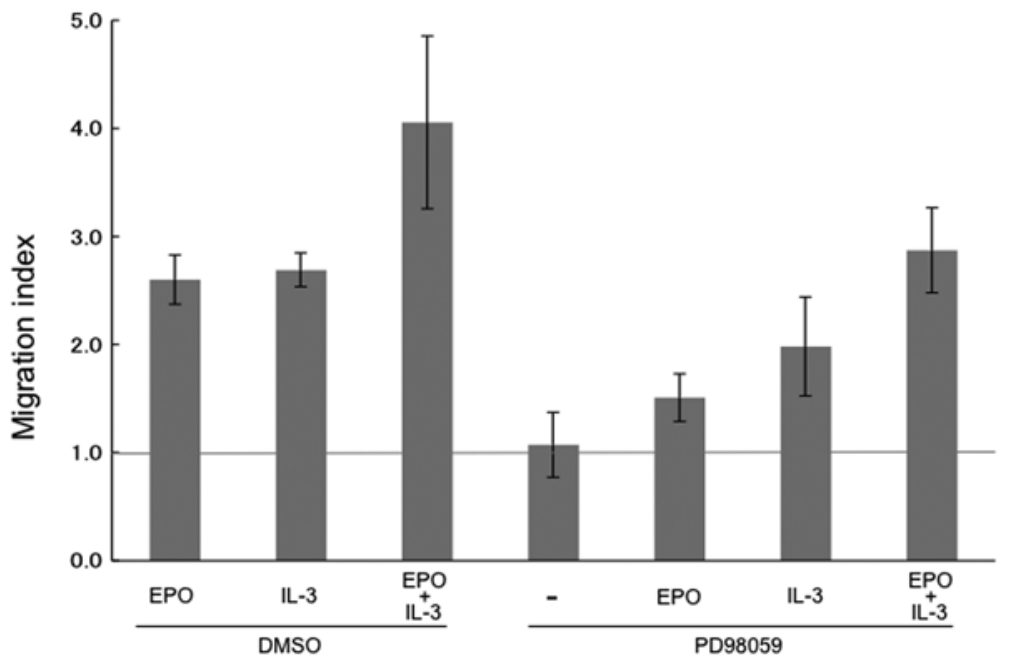

Figure 8. Effect of EPO on the induction of spontaneous migration. EPOR/CR3 cells were used for Transwell migration assays and the number of viable cells in the lower chamber was determined after $5 \mathrm{~h}$ by trypan blue exclusion. Vertical axis; migration index expressed as the ratio of the number of either EPO and/or IL-3 stimulated cells with DMSO $(0.5 \%)$ or PD98059 $(50 \mu \mathrm{M})$ pretreatment migrating to the number of unstimulated cells with DMSO migrating over the same time period. PD98059 did not affect spontaneous migration over that of the untreated control.

The EPOR/CR3 protein, which was shown to have EPO-dependent Raf kinase activity, did not function in the absence of EPO, but could be reversibly activated by the addition or removal of EPO from the culture media. Thus, the EPOR/CR3 chimeric receptor provides a method to rapidly and reversibly dimerize the Raf protein to induce its activity. Other such techniques have been successfully used to activate
Raf kinase, generating either an hrafER (17) or GyrB-Raf1 (18) fusion protein, which was constructed by gene fusion between an oncogenic form of Raf and a hormone-binding domain of the human estrogen receptor (ER) or gyrase B (GyrB), respectively. Previous studies have shown that Raf kinase is induced in both chimeric proteins, which can be artificially dimerized by the addition of their ligand, estradiol, or the antibiotic coumer- 
mycin to the culture media and with the known transforming activity of v-raf. Therefore, it is clear that the dimerization or aggregation of Raf is important for its activation, as observed in this study. Nevertheless, the most prominent difference in EPOR/CR3 from these chimeric molecules may be in the subcellular localization of the fusion proteins. The subcellular localization of the former chimeric proteins, where kinase activity is artificially induced, has not been clearly demonstrated, but is likely to be in a different cellular compartment, with gyrase B presumably being a nuclear protein, the estrogen receptor being detected both in the nucleus and cytoplasm, and Raf being primarily, if not exclusively, cytoplasmic.

On the other hand, the EPOR/CR3 receptor reported here is of particular interest because the oncogenic form of Raf, lacking the Ras binding domain, is tethered to the cell membrane where endogenous Raf is normally activated by binding with Ras. This is especially important in evaluating the signaling cascade that originates from molecules.

This new model also demonstrates that the recruitment of Raf to the plasma membrane itself is not sufficient to activate Raf kinase activity to exhibit the biochemical and biological properties of EPOR/CR3, in contrast to a previous report suggesting that the targeting of Raf to the plasma membrane leads to its activation (19).

When substrates phosphorylated in EPOR/CR3 Ba/f3 cells induced by either EPO or IL-3 were compared, EPO was shown to induce minimum tyrosine phosphorylation of the signaling molecules against that of IL-3 phosphorylates. The addition of EPO leads to the rapid phosphorylation of ERK in cells, which is a well known substrate of Raf, indicating that Raf can be activated by a mechanism that induces clustering at the cell membrane independent of other upstream factors, and that this leads directly to the activation of MEK and ERK. On the other hand, unlike IL-3, the stimulation of EPO did not induce the tyrosine phosphorylation of STAT and PI3K, indicating that neither the STAT nor PI3K pathway was activated by Raf kinase. Several other cellular proteins, mostly unknown, are also phosphorylated on tyrosine upon EPO stimulation. As shown in this study, some of this phosphorylation was not abolished by PD98059, which suggests that any protein tyrosine kinase besides ERK may be involved in the cascade. In addition, the inducible activation of EPOR/CR3 in this mutant line restored dual ERK phosphorylation and the mobility shift of endogenous Raf-1, indicating that this shift was due to ERK mediated feedback phosphorylation and that these two events were connected to each other. The potential problem of the chimeric receptor is that the protein is fixed to the cell membrane constitutively, therefore, it does not reflect the natural dynamics of Raf once this is activated. Because of this difference in dynamics, stimulating regulation with growth factors that normally induce Raf function by changing the cellular localization may have different effects on this chimeric molecule, such as protein interactions and/or cellular outcomes. It is possible that although EPOR/CR3 alone is incomplete, with the help of endogenous Raf-1 presumably changing the cellular localization, it can induce the essential signals and biological properties of this system.

The biological effects of Raf kinase, when activated as part of the EPOR/CR3, were focused on in order to estimate its contribution to the etiology of human cancer. This is of particular interest not only to understand the molecular mechanism of the disorders, but also to develop Raf inhibitors as an attractive targeted therapy. Small molecule drugs that can selectively inhibit kinases are likely to be of benefit in a number of neoplastic diseases. Among the drugs invented, BAY 43-9006 was the first orally bioavailable multikinase inhibitor that targets Raf kinase to undergo clinical testing (20). In the present study, we sought to directly address this question by determining whether the EPOR/CR3 fusion protein had in vitro transforming activity. As presented here, EPO-induced activation of Raf kinase can lead to dose-dependent increases in viability, mobility, and short-term cell proliferation in EPOR/CR3 Ba/F3 cells. However, the level of proliferation by EPO was lower than that observed in the case of IL-3 stimulation, and proliferation was only short-term. The biological effects of activating the EPOR/CR3 in Ba/F3 cells are striking changes in viability, hyper-responsiveness to growth factors, and enhanced migration. Activated Raf can add to suboptimal growth signals from another receptor and this could be one of the most important components of the transforming activity of the Raf oncogene.

Recently, over 30 mutations in the B-Raf gene have been associated with human cancers, the majority of which are located within the kinase domain (10). Although it is still incompletely understood whether a sole change is sufficient to induce cancer in humans, results suggest that the inhibition of kinase activity may be an important new strategy in the treatment of sporadic tumors. There is a medical need for the development of excellent model systems, in which the biochemical and biological effects of Raf and the inhibitory effect of its activity can be studied, in order to identify effective therapies. The chimeric receptor reported here will facilitate in vitro signaling studies of Raf as the kinase is rapidly turned on and off, and will enable the effects of signal transduction inhibitors of Raf as a target to be evaluated. Moreover, the receptor has some interesting features that may be useful for better understanding the function of the Raf oncogene, particularly because of its in vivo applications.

Future efforts to generate an in vivo mouse model of inducible cancer, in which the activity of Raf can be turned on and off with an exogenous ligand, would be of enormous value to the field. The rapid activation of a kinase cascade by the EPOR/CR3 fusion protein clearly demonstrates the utility of this approach and will be useful in the identification of new substrates of Raf kinase, better understanding of the pathogenesis in the Raf oncogene, and should lead to new treatments for the disease.

\section{Acknowledgements}

The author would like to thank Dr James D. Griffin, Dana-Farber Cancer Institute, USA, for his great encouragement and helpful discussions in carrying out this study. This study was supported in part by a Grant-in-Aid for Scientific Research from the Ministry of Education, Culture, Sports, Science and Technology of Japan, and a Research Fund of the Astellas Foundation for Research on Metabolic Disorders.

\section{References}

1. Morrison DK: The Raf-1 kinase as a transducer of mitogenic signals. Cancer Cells 2: 377-382, 1990.

2. Rapp UR: Role of Raf-1 serine/threonine protein kinase in growth factor signal transduction. Oncogene 6: 495-500, 1991. 
3. Moodie SA, Willumsen BM, Weber MJ and Wolfman A Complexes of Ras-GTP with Raf-1 and mitogen-activated protein kinase. Science 260: 1658-1661, 1993.

4. Morrison DK and Cutler RE: The complexity of Raf-1 regulation. Curr Opin Cell Biol 9: 174-179, 1997.

5. Vojtek AB, Hollenbeg SM and Cooper JA: Mammalian ras interacts directly with the serine/threonine kinase Raf. Cell 74 205-214, 1993.

6. Zimmermann S and Moelling K: Phosphorylation and regulation of Raf by Akt (protein kinase B). Science 286: 1741-1744 1999.

7. Stanton VJ, Nichols DW, Laudano AP and Cooper GM: Definition of the human raf amino-terminal regulatory region by deletion mutagenesis. Mol Cell Biol 9: 639-647, 1989.

8. Dent P, Haser W, Haystead TAJ, Vincent LA, Roberts TM and Sturgill TW: Activation of mitogen-activated protein kinase kinase by v-Raf in NIH3T3 cells and in vitro. Science 257 1404-1407, 1992.

9. Cleveland JL, Troppmair J, Packham G, Askew DS, Lloyd P, Gonzalez-Garcia M, Nunez H, Ihle JN and Rapp UR: $\mathrm{V}$-raf suppresses apoptosis and promotes growth of interleukin-3-dependent myeloid cells. Oncogene 9: 2227-2233, 1994.

10. Davies H, Bignell GR, Cox C, Stephens P, Edkins S, Clegg S, Teague J, Woffendin H, Garnett MJ, Bottomley W, Davis N, Dicks E, Ewing R, Floyd Y, Gray K, Hall S, Hawes R, Hughes J, Kosmidou V, Menzies A, Mould C, Parker A, Stevens C, Watt S, Hooper S, Wilson R, Jayatilake H, Gusterson BA, Cooper C, Shipley J, Hargrave D, Pritchard-Jones K, Maitland N, Chenevix-Trench G, Riggins GJ, Bigner DD, Palmieri G, Cossu A, Flanagan A, Nicholson A, Ho JWC, Leung SY, Yuen ST, Weber BL, Seigler HF, Darrow TL, Paterson H, Marais R, Marshall CJ, Wooster R, Stratton MR and Futreal PA: Mutation of the BRAF gene in human cancer Nature 417: 949-954, 2002

11. Ciampi R, Knauf JA, Kerler R, Gandhi M, Zhu Z, Nikiforova MN, Rabes HM, Fagin JA and Nikiforov YE: Oncogenic AKAP9-BRAF fusion is a novel mechanism of MAPK pathway activation in thyroid cancer. J Clin Invest 115: 94-101, 2005.
12. Wan PTC, Garnett MJ, Roe SM, Lee S, Niculescu-Duvaz D, Good VM, Jones CM, Marshall CJ, Springer CJ, Barford D and Marais R: Mechanism of activation of the RAF-ERK signaling pathway by oncogenic mutation of B-RAF. Cell 116: 855-867, 2004.

13. Okuda K, D'Andrea A, Van Etten RA and Griffin JD: A chimeric receptor/oncogene that can be regulated by a ligand in vitro and in vivo. J Clin Invest 100: 1708-1715, 1997.

14. Sternberg PW and Alberola-Ila J: Conspiracy theory: Ras and Raf do not act alone. Cell 95: 447-450, 1998.

15. D'Andrea AD, Yoshimura A, Youssoufian H, Son LI, Koo JW and Lodish HF: The cytoplasmic region of the erythropoietin receptor contains non-overlapping positive and negative growth-regulatory domains. Mol Cell Biol 11: 1980-1987, 1991.

16. Barber DE, DeMartino JC, Showers MO and D'Andrea AD: A dominant negative erythropoietin (EPO) receptor inhibits EPO-dependent growth and blocks F-gp55-dependent transformation. Mol Cell Biol 14: 2257-2265, 1994.

17. Samuels ML, Weber MJ, Bishop JM and McMahon M Conditional transformation of cells and rapid activation of the mitogen-activated protein kinase cascade by an estradioldependent human Raf-1 protein kinase. Mol Cell Biol 13: 6241-6252, 1993

18. Farrar MA, Alberola-Ila J and Perlmutter RM: Activation of the raf-1 kinase cascade by coumermycin-induced dimerization. Nature 383: 178-181, 1996.

19. Leevers SJ, Paterson HF and Marshall CJ: Requirement for Ras in Raf activation is overcome by targeting Raf to the plasma membrane. Nature 369: 411-414, 1994.

20. Wilhelm SM, CarterC, Tang L, Wilkie D, McNabola A, Rong H, Chen C, Zhang X, Vincent P, McHugh M, Cao Y, Shujath J, Gawlak S, Eveleigh D, Rowley B, Leu L, Adnane L, Lynch M, Auclair D, Taylor I, Gedrich R, Voznesensky A, Riedl B, Post LE, Bollag G and Trail PA: BAY 43-9006 exhibits broad spectrum oral antitumor activity and targets the RAF/MEK/ERK pathway and receptor tyrosine kinases involved in tumor progression and angiogenesis. Cancer Res 64: 7099-7109, 2004. 